\title{
Korean version of pain-related self-report questionnaires
}

\author{
Jong-Man Kang
}

Department of Anesthesiology and Pain Medicine, Kyung Hee University, Kyung Hee University Hospital at Gangdong, Seoul, Korea

Pain is one of the most common reasons that people visit medical care facilities. It is defined as an unpleasant sensory and emotional experience associated with actual or potential tissue damage, or is described in terms of such damage [1]. Pain is considered a purely subjective experience, but its management should be objective. Pain assessment is the first step in optimal pain management.

Pain assessment should begin with an open-ended statement or question such as "Tell me about your pain" or "What does your pain feel like?" The pain intensity, location, duration, and aggravating or alleviating factors should be described. We tend to use only a visual analog scale or numeric pain rating scale to manage patients' pain and for convenience when performing clinical studies on pain. However, these scales only assess the intensity of pain; they do not address any other components of the pain experience.

Pain, especially chronic pain, evokes other general and psychosocial health problems. When a physician treats or manages a patient with chronic pain, he or she should also be concerned about the overall health of the patient. Patient-oriented measurement instruments have been developed to measure these pertinent details using questionnaires that can be answered by the patients themselves. The 36-Item Short Form Health Survey, Beck Depression Inventory, Beck Anxiety Inventory, Oswestry Disability Index, and Pittsburgh Sleep Quality Index have been used to evaluate quality of life, depression, anxiety, disability, and sleep disturbances of patients and were chosen for the patient with chronic low back pain in this month's study [2]. A Korean version of these instruments would be helpful in every clinic of Korea to manage patients with chronic pain. The simple literacy rate of Korea is very high; however, the Programme for the International Assessment of Adult Competencies showed that Korea is among the three lowest-performing countries in the Organisation for Economic Co-operation and Development (OECD) with respect to the skill proficiency of 55- to 65-yearolds. On the other hand, Korea ranks second only to Japan in terms of proficiency among 16- to 24-year-olds [3]. Thus, Korean versions should be made to be easily understandable by all patients, especially the elderly. Moreover, various modifications of currently available versions (subversions) should be established according to differences in dialect, sex, generation, and level of education. Patients may find it difficult or be reluctant to properly describe their health state, pain severity, and other associated problems. While completing the questionnaire, patients have the opportunity to comprehensively assess and understand their health status. In very busy outpatient clinics in Korea, there is often inadequate time for communication between patients and physicians. Self-reporting may save consultation time if performed at home or before seeing a doctor.

Korean physicians may experience conflict in choosing between the Korean and English language when writing medical records during communication with patients because each language has its own merits and limitations. Documentation in Korean allows the doctor to easily describe the patient's self-report as it was stated and to recall the status of the patient during later visits. However, it can induce confusion in terminology with today's highly globalized medical community. If standard Korean assessment instruments for pain and its associated health status, symptoms, and problems are made to be easily "intertranslated" between Korean and English and are distributed in every clinic,

Corresponding author: Jong-Man Kang, M.D., Ph.D., Department of Anesthesiology and Pain Medicine, Kyung Hee University, Kyung Hee University Hospital at Gangdong, 892, Dongnam-ro, Gangdong-gu, Seoul 134-090, Korea. Tel: 82-2-440-6193, Fax: 82-2-440-7808, E-mail: jongmankang@gmail.com

(c) This is an open-access article distributed under the terms of the Creative Commons Attribution Non-Commercial License (http:// creativecommons.org/licenses/by-nc/3.0/), which permits unrestricted non-commercial use, distribution, and reproduction in any medium, provided the original work is properly cited. 
communication with patients would be easier. Moreover, this would facilitate discussions with other health providers regarding pain management and allow for the performance of clinical investigations in an internationally standardized style.

We often hear complaints of back pain after spinal operations, but we do not usually obtain information about the preoperative pain assessment results from the medical record. This prevents comparison of the pre- and postoperative pain characteristics despite the fact that the operation was performed in the same hospital. Pain assessment should occur at regular intervals, be individualized, and be documented and easily understood by all medical staff members involved to most appropriately manage the patient's pain. Regardless of the level of qualification and organization of any assessment tool, the clinician should listen, understand, believe, and sympathize with the patient as a human being. This attitude can be very effective for optimal pain management.

\section{References}

1. Pain terms: a list with definitions and notes on usage. Recommended by the IASP Subcommittee on Taxonomy. Pain 1979; 6: 249.

2. Hong JH, Kim HD, Shin HH, Huh B. Assessment of depression, anxiety, sleep disturbance, and quality of life in patients with chronic low back pain in Korea. Korean J Anesthesiol 2014; 66: 444-50.

3. Skilled for Life? Key findings from the survey of adult skills. OECD Skills Outlook 2013: 12. 